\title{
Interleukin-18, interleukin-20, and matrix metalloproteinases (MMP-1, MMP-3) as markers of psoriatic arthritis disease severity and their correlations with biomarkers of inflammation and turnover of joint cartilage
}

Michał Waszczykowski ${ }^{1}$, Igor Bednarski ${ }^{2}$, Joanna Narbutt ${ }^{2}$, Elżbieta Waszczykowska ${ }^{3}$, Aleksandra Lesiak ${ }^{2}$, Jarosław Fabiśs

\author{
1Department of Arthroscopy, Minimally Invasive Surgery, and Sports Traumatology, Medical University of Lodz, Lodz, Poland \\ 2Dermatology, Paediatric Dermatology, and Oncology Clinic, Medical University of Lodz, Lodz, Poland \\ ${ }^{3}$ Department of Dermatology and Venereology, Medical University of Lodz, Lodz, Poland
}

Adv Dermatol Allergol 2020; XXXVII (6): 1001-1008

DOI: https://doi.org/10.5114/ada.2020.94903

\begin{abstract}
Introduction: Psoriatic arthritis (PsA) is a chronic, seronegative spondyloarthropathy characterised by joint inflammation and psoriatic skin changes. Recent data indicate that interleukin-18 (IL-18) and interleukin-20 (IL-20) may be involved in the aetiopathogenesis of PsA.

Aim: To evaluate the potential role of IL-18, IL-20, and matrix metalloproteinases (MMP-1, MMP-3) in the pathogenesis of PsA and their correlations with other markers of inflammation and destruction of joint cartilage, as well as clinical changes.

Material and methods: The study included 24 patients with PsA and 26 healthy volunteers as a control group. The concentration of IL-18 and IL-20, c-reactive protein (CRP), metalloproteinase-1 and -3 (MMP-1, MMP-3), cartilage oligomeric matrix protein (COMP), aggrecan (PG-AG), and human cartilage glycoprotein (YKL-40) in serum was determined. Clinical severity of the disease according to the BSA, PASI, and DLQI as well as tender and swollen joint count (TJC, SJC) were also evaluated.

Results: The concentration of IL-18 was statistically significantly higher in the serum of patients with PsA than in the control group (62.87 pg/ml vs. $16.73 \mathrm{pg} / \mathrm{ml}, p<0.0049)$. Serum IL-20 levels in PsA patients were also higher than in the control group, but without statistical significance $(p=0.2939)$. The ROC curves showed: AUC $=0.81$ for IL-18, $\mathrm{AUC}=0.75$ for IL-20, AUC $=0.96$ for COMP, and $\mathrm{AUC}=0.89$ for MMP-3.

Conclusions: IL-18 and IL-20 as well as MMP-3 and COMP may be sensitive markers in the diagnosis of PsA.
\end{abstract}

Key words: interleukin-18, interleukin-20, matrix metalloproteinases, cartilage oligomeric matrix protein, psoriatic arthritis.

\section{Introduction}

Psoriatic arthritis (PSA) is a chronic autoimmune disease characterised by joint inflammation and psoriatic skin changes [1, 2]. PsA occurs in about $30 \%$ of patients with psoriasis, which in about $85 \%$ of cases precedes or occurs in parallel with PsA [2, 3]. Its prevalence is estimated at about $0.2 \%$ of the total population, but its course and consequences may lead to significant impairment of the skeletal system and a significant reduction in quality of life at an early age [2, 4, 5]. People with PsA are at increased risk of cardiovascular diseases, metabolic diseases, and obesity [6]. The aetiopathogenesis of PsA is a complex multi-factor process. Although numerous studies on PsA aetiopathogenesis have been conducted for many years, it has not been possible to establish its full course and all metabolic, biochemical, and immunoenzymatic pathways and processes [1-7]. In recent years research has contributed to great progress in the knowledge of the mediators involved in the pathogenesis of PsA. However, it still seems that we do not know the

Address for correspondence: Michał Waszczykowski MD, PhD, Department of Arthroscopy, Minimally Invasive Surgery, and Sports Traumatology, Medical University of Lodz, 4 Kosciuszki St, 90-419 Lodz, Poland, phone: +48 693865 999, e-mail: mwaszczykowski@wp.pl Received: 29.03.2020, accepted: 2.04.2020. 
full list of PsA mediators. Better understanding of the pathogenesis of this disease may allow earlier detection, more effective treatment, and prevention of irreversible complications.

Studies in recent years have shown that interleukin-18 (IL-18) and interleukin-20 (IL-20) may be involved in the aetiopathogenesis of PSA, and that biological treatment may have an inhibitory effect on their activity [8, 9].

Interleukin 18 is part of the interleukin 1 (IL-1) superfamily and is mainly produced by macrophages, osteoblasts, keratinocytes, chondrocytes, dendritic cells, and neutrophils. It is formed as an inactive form of the $23 \mathrm{kDa}$ protein to be activated to the $18 \mathrm{kDa}$ protein form by the action of the proteolytic enzyme caspase 1 [10, 11]. Its main action is to intensify chemotaxis of $T$ cells and stimulate their differentiation to the helper cells Th1 and Th2. Interleukin 18 also stimulates neutrophil and monocyte chemotaxis, and enhances the cytotoxicity of NK cells and the secretion of interferon $\gamma$. Another important role of this cytokine is activation and stimulation of macrophages for secretion of tumour necrosis factor $\alpha$ (TNF- $\alpha$ ) and interleukin $1 \beta$ (IL-1 $\beta$ ), which affects the stimulation of the inflammatory response and increases the destruction of articular cartilage. The action of IL-18 on fibroblasts increases the expression of vascular endothelial growth factor (VEGF), chemokines, IL-8/CXCL8, intercellular adhesion molecule 1 (ICAM-1), and vascular cell adhesion molecule 1 (VCAM-1), which increases the inflammatory response and intensifies angiogenesis [10, 11]. It seems that IL-18, after IL-1 $\beta$, may be a key cytokine mediating the inflammatory response of the synovial membrane, intensifying expression of metalloproteinases and IL-6, and stimulating the destruction of aggrecan and the extracellular matrix [12-14]. Moreover, recent studies indicate that IL-18 may inhibit the synthesis of proteoglycans, aggrecan, and type II collagen in chondrocytes, initiate apoptosis of the chondrocytes themselves, and, through a positive effect on angiogenesis, intensify the processes of joint cartilage destruction [11, 13, 15].

The IL-20 belongs to the IL-10 family, which includes: IL-10, IL-19, IL-20, IL-22, IL-24, and IL-26. It has a complex of two receptors: IL-20R1/IL-20R2 and IL-22R1/IL-20R2 $[16,17]$. IL-20 and its receptors are expressed in several normal tissue types, including skin, kidney, and lungs $[16,18]$. Recent reports have demonstrated its role in the aetiopathogenesis of rheumatoid arthritis (RA), systemic sclerosis, atherosclerosis, myocardial infarction, stroke, prostate cancer, renal disorders, and osteoarthritis [1928]. Recent studies have also shown that IL-20 plays an important role as a proinflammatory cytokine in psoriasis $[29,30]$. IL-20 expression was elevated in lesional psoriatic skin compared with normal skin [28, 31, 32]. IL-20 is a cytokine with chemotactic and proinflammatory effects, which positively influences angiogenesis and apoptosis, affecting fibroblasts of the synovial membrane, endothelial cells, keratinocytes, and kidney epithelial cells
$[16,23,28]$. This interleukin increases also the migration of neutrophils and T cells [20]. IL-20 is secreted mainly by fibroblasts and macrophages granulocytes but also by keratinocytes (KCS), maturing dendritic cells (DCs), endothelial cells, and renal mesangial cells $[17,19,20]$. A recent report shows its stimulating effect on TNF- $\alpha$, IL-1 $\beta$, and metalloproteinase 1 and 13 (MMP-1 and MMP-13) secretion in osteoarthritic synovial fibroblasts (OASFs) [17]. IL-20 affects chondrocytes by increasing the activity of interleukin 6 (IL-6) and MMPs, but also inhibits the expression of transforming growth factor $\beta$ (TGF- $\beta$ ) and the ability to synthesise aggrecan [17].

\section{Aim}

There are few data on the contribution of these interleukins to the aetiopathogenesis of PsA and their role in the transformation and destruction of joint cartilage. Recent studies by Hsu and Chang, Scrivo et al., and Przepiera-Będzak et al. show the elevated concentration of IL-18 and IL-20 in serum and synovial tissue, indicating that these interleukins may play an important role in the pathogenesis of PsA $[8,9,20]$. The aim of this study was to assess the potential role that IL-18, IL-20, and matrix metalloproteinases (MMP-1, MMP-3) play in the pathogenesis of PsA, by evaluating their serum concentration and their correlation with the presence of other proinflammatory markers and cytokines in serum: C-reactive protein (CRP), erythrocyte sedimentation rate (ESR) and markers of joint cartilage destruction: cartilage oligomeric matrix protein (COMP), human cartilage glycoprotein (YKL-40), aggrecan (PG-AG), as well as the clinical stage of disease expressed by body surface areas score (BSA), Psoriasis Area and Severity Index (PASI), Dermatology Life Quality Index (DLQI), and the number of painful (TJC) and swollen joints (SJC) [33-36].

\section{Material and methods}

\section{Study group}

Patients were qualified for the study at the Department of Dermatology and Venereology of the Medical University of Lodz and the Orthopaedic Outpatient Clinic operating at the clinic. Based on the clinical examination and the Classification of Psoriatic Arthritis Study Group (CASPAR) criteria, the diagnosis of PsA was made [37]. In all patients CRP and ESP were determined in peripheral blood serum. Radiological imaging or additional laboratory tests were performed only when needed. The previous analysis excluded patients with a history of past or present other immunological diseases (RA, Crohn's disease), neoplastic diseases, and acute or past significant injuries. Only patients with active disease status, who did not react to the previous standard of disease-modifying anti-rheumatic drugs (DMARDs) and were not treated previously with anti-TNF drugs were qualified for the 
study. The control group consisted of healthy volunteers corresponding to age and gender distribution for the study group, who did not show features of psoriasis and arthritis. All patients and control subjects gave informed and written consent to participate in the study. The local Bioethics Committee accepted and agreed to the study (consent number: RNN/36/06/KB), which was performed in accordance with the Helsinki Declaration.

\section{Clinical assessment}

The BSA, PASI, and DLQI were used in the clinical assessment of patients and the severity of the disease [33-36]. For clinical assessment and joint involvement we used a 68 tender and 66 swollen joint count (TJC, SJC), including the distal interphalangeal (DIP) joints of the hands and excluding hips for swelling [38].

\section{Determination of level of IL-18 and IL-20 and other markers in serum}

Subsequently, IL-18, IL-20, MMP-1, MMP-3, COMP, YKL-40, and PG-AG serum concentration measurements were performed with enzyme-linked immunosorbent assay (ELISA) kits in the study and control groups. Peripheral blood samples were taken from each patient in the morning, then centrifuged, and the obtained serum was deposited into $1.5 \mathrm{ml}$ Eppendorfs, which were then sealed, frozen, and stored at $-80^{\circ} \mathrm{C}$ for further immunoenzymatic determinations. Then, measurements of serum concentrations of IL-18, IL-20, MMP-1, MMP-3, COMP, YKL-40, and PG-AG were performed using ELISA - enzyme-linked immunosorbent assay sets from R\&D Systems Europe, Ltd, Abingdon, UK (IL-18, IL-20, MMP-1, MMP-3), BioVendor GmbH, Heidelberg, Germany (COMP), Metra Quidel, San Diego, USA (YKL-40), BioSource Europe S.A., Nivelles, Belgium (PG-EASIA), according to the manufacturers' instructions. A minimum detection level was set: $4.57 \mathrm{pg} / \mathrm{ml}$ for IL-18, $16.6 \mathrm{pg} / \mathrm{ml}$ for IL-20, 0.095 $\mathrm{ng} / \mathrm{ml}$ for MMP-1, 0.045ng/ml for MMP-3, $0.4 \mathrm{ng} / \mathrm{ml}$ for COMP, $10 \mathrm{ng} / \mathrm{ml}$ for YKL-40, and $0.9 \mathrm{ng} / \mathrm{ml}$ for PG-EASIA.

The same activities (blood collection, centrifugation, freezing, and storage) were performed in the control group, followed by the same immunoentimacy determinations as in the study group using appropriate ELISA kits (R\&D Systems, BioVendor, Metra Quidel, BioSource). Due to technical reasons the PG-AG concentration was not determined in the control group.

\section{Statistical analysis}

Baseline characteristics of participants including age, clinical scales, and laboratory findings were presented as means with standard deviations or medians with interquartile range. Distribution of variables was evaluated using the Shapiro-Wilk test. Due to non-Gaussian distribution of variables the Mann-Whitney $U$ test was used to compare differences between the control and study group. To compare differences between two dependent variables the Wilcoxon signed rank test was employed. Friedman's ANOVA was used to calculate differences between more than two dependent variables. Correlations between each variable were examined using Spearman's rank correlation. A correlation coefficient ranging 0.00 to 0.19 was considered as very weak, 0.20 to 0.39 as weak, 0.40 to 0.59 as moderate, 0.60 to 0.79 as strong, and 0.80 to 1.0 as very strong.

A p-value below 0.05 was considered statistically significant. The diagnostic performance of each biomarker in PsA detection was assessed by ROC curve analysis with calculation of Youden's Index and the area under the curve (AUC). All calculations were made using Statistica 13 software.

\section{Results}

Finally, 24 patients ( $57 \%$ of them were men) aged between 32 and 71 years (mean: 54.18 years) were qualified for the analysis. The control group consisted of 26 healthy volunteers. There were no statistically significant differences between the two groups in terms of age and gender (Table 1). We found a statistically significant increase in serum levels of IL-18 in the examined patients with PsA compared to the control group ( $p<0.005$; Table 1 ). Serum IL-20 levels in PSA patients were also higher than in the control group, but this increase was not statistically significant ( $p=0.2939$; Table 1$)$. We also found

Table 1. Clinical parameters and serum concentrations of metalloproteinases, inflammatory cytokines, and cartilage turnover markers in the study (PSA) and control groups

\begin{tabular}{|c|c|c|c|}
\hline Parameter & $\operatorname{PsA}(N=24)$ & Control $(N=26)$ & $P$-value \\
\hline Mean age [years] & $54.18 \pm 9.35$ & $54.58 \pm 8.92$ & 0.8229 \\
\hline MMP-1 [ng/ml] ${ }^{*}$ & $4.40 \pm 2.37$ & $7.27 \pm 3.78$ & $<0.005$ \\
\hline MMP-3 $[\mathrm{ng} / \mathrm{ml}]$ * & $22.23 \pm 19.25$ & $9.08 \pm 3.13$ & $<0.0001$ \\
\hline IL-18 [pg/ml]* & $62.87 \pm 66.87$ & $16.73 \pm 17.00$ & $<0.005$ \\
\hline $\mathrm{IL}-20[\mathrm{pg} / \mathrm{ml}]^{\star}$ & $12.98 \pm 12.30$ & $9.76 \pm 9.00$ & 0.2939 \\
\hline YKL-40 [ng/ml]* & $75.04 \pm 37.51$ & $44.95 \pm 19.19$ & 0.0061 \\
\hline $\operatorname{COMP}[\mathrm{ng} / \mathrm{ml}]^{*}$ & $2645.26 \pm 489.48$ & $835.85 \pm 434.62$ & $<0.0001$ \\
\hline PG-AG $[\mathrm{ng} / \mathrm{ml}]^{\star}$ & $3878.46 \pm 617.93$ & - & \\
\hline PASI $(\%)^{*}$ & $16.53 \pm 3.93$ & - & \\
\hline $\mathrm{DLQI}^{*}$ & $13.87 \pm 2.13$ & - & \\
\hline $\mathrm{BSA}(\%)^{*}$ & $26.47 \pm 8.41$ & - & \\
\hline $\mathrm{CRP}[\mathrm{mg} / \mathrm{l}]^{\star}$ & $9.48 \pm 8.54$ & - & \\
\hline $\mathrm{ESR}[\mathrm{mm} / \mathrm{h}]^{\star}$ & $12.75 \pm 7.44$ & - & \\
\hline $\operatorname{TJC}(0-68)^{\star}$ & $5.75 \pm 1.53$ & & \\
\hline $\operatorname{SJC}(0-66)^{*}$ & $4.62 \pm 0.96$ & & \\
\hline $\mathrm{TJC}+\mathrm{SJC}^{*}$ & $10.56 \pm 2.53$ & & \\
\hline
\end{tabular}

*Values given as mean with standard deviation. 
Table 2. Significant Spearman's correlation coefficients between studied clinical parameters and inflammatory/ cartilage biomarkers in serum

\begin{tabular}{lcc}
\hline Pair of parameters $(\boldsymbol{N}=\mathbf{2 4})$ & $\boldsymbol{R}$ & $\boldsymbol{P}$-value \\
\hline MMP-1 vs. PASI & 0.5993 & 0.0182 \\
\hline MMP-1 vs. DLQI & 0.5300 & 0.0421 \\
\hline IL-20 vs. BSA & 0.7096 & 0.0097 \\
\hline COMP vs. PASI & 0.7311 & 0.0020 \\
\hline COMP vs. DLQI & 0.5559 & 0.0314 \\
\hline COMP vs. SJC & 0.6399 & 0.0076 \\
\hline COMP vs. TJC + SJC & 0.5886 & 0.0165 \\
\hline
\end{tabular}

that MMP-3 showed a statistically significant higher serum level in PsA patients compared to the control group ( $p<0.0001$; Table 1). Surprisingly, we found that the concentration of MMP-1 in serum PsA patients was statistically significant lower than in the control group $(p<0.005$; Table 1).

In our study we also analysed the concentrations of markers of turnover and destruction of joint cartilage in the serum of patients with PsA and compared them with their level in the control group. We showed that both YKL-40 and COMP had significantly higher serum concentrations in the study group compared to controls $(p=0.0061$ for $Y K L-40$ and $p<0.0001$ for COMP, respectively; Table 2).

In our study we did not find any strong, significant correlations between the IL-18 and IL-20 and any other studied markers of inflammation and cartilage destruction in serum in PsA patients. However, we indicated positive correlation between IL-20 level in serum in PsA patients and degree of clinical severity of the disease (BSA score; Table 2).

Our data also indicated that serum MMP-1 levels in the PsA study group positively correlated with the degree of clinical severity of the disease on both the PASI and DLQI scales (Table 2). Moreover, our analysis also showed strong correlations between one of the markers of cartilage destruction in serum in PSA patients (COMP) and the clinical and functional stage of PSA (PASI, DLQI, TJC, SJC; Table 2). We also found strong correlation between COMP serum concentration in PSA patients and degree of joint involvement expressed in SJC, and in SJC and TJC together (Table 2).

To estimate the diagnostic performance of each biomarker we decided to employ ROC curve analysis with Youden's index. Almost all (except YKL-40) biomarkers showed significant predictive value in diagnosing PsA: MMP-1 (AUC = 0.76, cut-off value $5.90 \mathrm{ng} / \mathrm{ml}), \mathrm{MMP}-3$ $(A \cup C=0.89$, cut-off value $14.30 \mathrm{ng} / \mathrm{ml}), \mathrm{IL}-18(\mathrm{AUC}=0.81$, cut-off value $40.18 \mathrm{pg} / \mathrm{ml}$ ), IL-20 (AUC $=0.75$, cut-off value $57.40 \mathrm{pg} / \mathrm{ml}$ ), and COMP (AUC = 0.96, cut-off value 1935 ng/ml) (Table 3; Figures 1-3).

\section{Discussion}

There are still few data in the literature on the contribution of IL-18 and IL-20 to the aetiopathogenesis of PsA. The available data mainly describe the contribution of these interleukins to the changes and processes occurring in other inflammatory arthropathies, especially in rheumatoid arthritis (RA), osteoarthritis (OA), or the cutaneous form of psoriasis [10, 12, 13, 20, 28, 39, 40]. In other studies, the compared groups were not homogeneous, and analysis of the results for the selected group of patients (PsA) is not possible [39]. Our analysis seems to be the first to attempt to show the potential impact of these interleukins on the pathogenesis and clinical aspect of PsA, their mutual correlations, and their common relationships with other markers of inflammatory process and cartilage destruction and the clinical evaluation of PsA patients.

The results of our study indicate significantly increased serum IL-18 levels in PSA patients compared to the control group. Similar observations were made by Rooney et al., who found a statistically significant increase in IL-18 levels in serum and synovium in PSA patients compared to the control group [41]. Similar results for serum IL-18 levels in PSA and other patients with inflammatory arthropathies (RA, AS, SnA, SAPHO syndrome), compared to the control group, were shown by Przepiera-Będzak et al. [9, 42]. Different results were obtained by Bresnihan et al. [43]; they found a marked increase in IL-18 levels in patients with RA compared to PsA. However, the study did not include a control group of healthy individuals.

Table 3. Receiver operating characteristic (ROC) data for serum concentrations of metalloproteinases, inflammatory cytokines, and cartilage turnover markers in the study (PsA) group

\begin{tabular}{lcccccc}
\hline Paramter & MMP-1 & MMP-3 & IL-18 & IL-20 & YKL-40 & COMP \\
\hline Cut-off value* $^{*}$ & 5.90 & 14.30 & 40.18 & 57.40 & 34.40 & 1935.00 \\
\hline Youden's Index & 0.54 & 0.69 & 0.57 & 0.52 & 0.26 & 0.96 \\
\hline AUC & 0.76 & 0.89 & 0.81 & 0.75 & 0.60 & 1.00 \\
\hline$P$-value & 0.0007 & 0.0000 & 0.0001 & 0.0039 & 0.2635 \\
\hline
\end{tabular}

*The values are given as $n g / m l$ (MMP-1, MMP-3, COMP, YKL-40) and $\mathrm{pg} / \mathrm{ml}(\mathrm{IL}-18, \mathrm{IL}-20)$. 

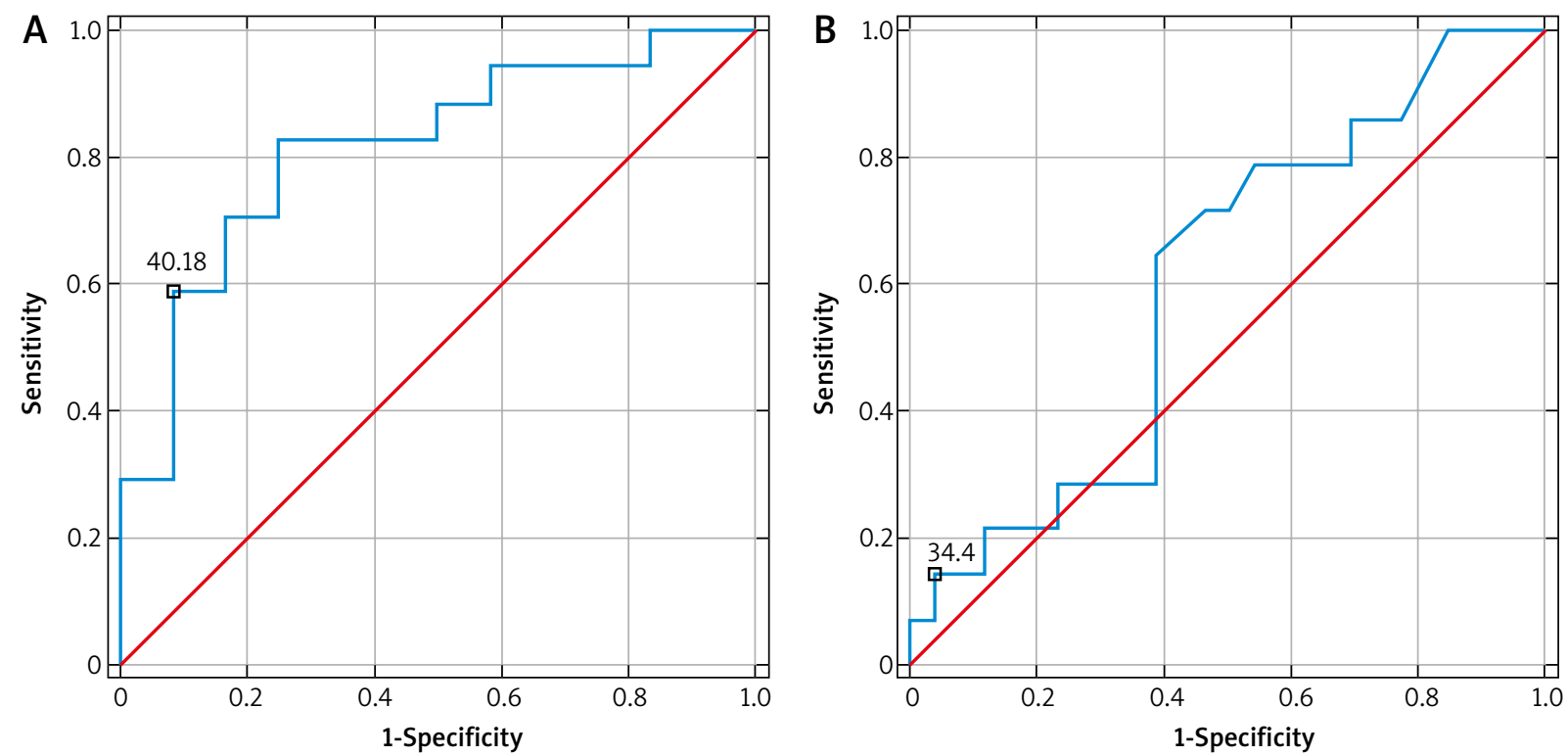

Figure 1. ROC curve analysis for: IL-18 (A) and IL-20 (B)

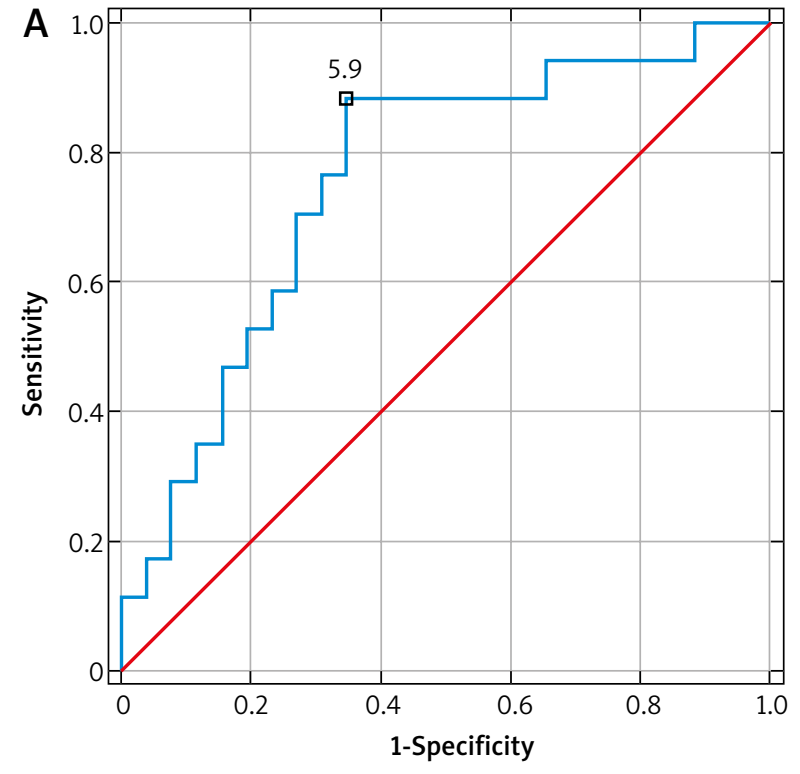

Figure 2. ROC curve analysis for: MMP-1 (A) and MMP-3 (B)

Our study also showed an increase in IL-20 serum levels in the examined patients (PsA) compared to the control group but without statistical significance. These results confirm the observations of Scrivo et al., who, in a fairly broad prospective study, showed statistically significantly elevated IL-20 levels in PsA patients compared to the control group [8]. Moreover, Scrivo et al. found that high serum IL-20 levels occur in both PSA and RA patients and are higher than in OA patients. Serum IL-20 levels among patients with PsA correlated positively with their synovial fluid levels.

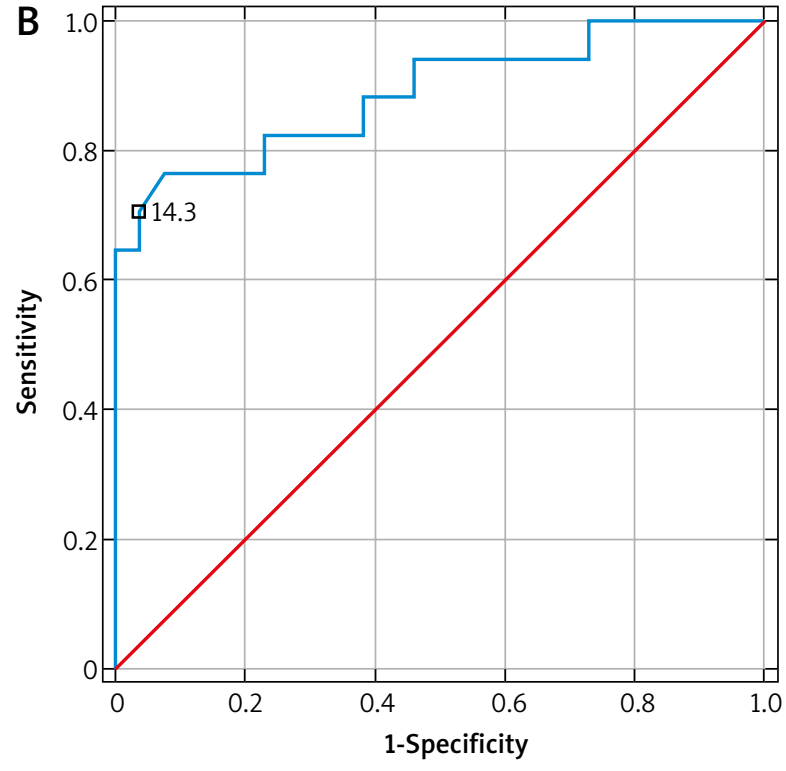

In our work we also tried to demonstrate whether the IL-18 and IL-20 levels correlate statistically significantly with clinical indicators of PsA disease progression. We used BSA, PASI, DLQI, tender joint count (TJC), and swollen joint count (SJC) grades to assess clinical changes. We only found that serum IL-20 levels positively correlated with the severity of skin lesions expressed by the BSA scale $(R=0.7096, p=0.00097$; Table 2). However, we were not able to indicate any other correlation between the concentration of the studied interleukins and the clinical severity of the disease. However, Przepiera- 


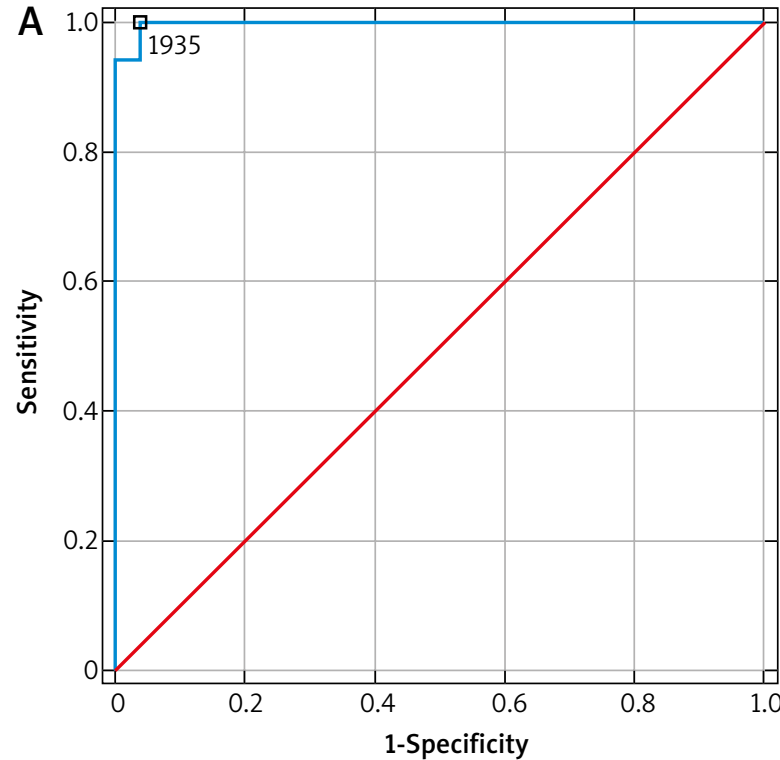

Figure 3. ROC curve analysis for: COMP (A) and YKL-40 (B)

Bedziak et al. in their paper demonstrated that IL-18 positively correlates only with the results of clinical assessment on the BASDAI scale $[9,42]$. However, Scrivo et al. did not demonstrate any correlation between the level of IL-20 and the clinical severity of PSA [8]. These observations may therefore indicate that although the activity of these interleukins is clearly elevated in patients with PsA, it may be primarily related to skin lesions and may not differentiate patients with psoriasis with articular lesions from those with pure skin form. In our analysis, we also pointed out the activity of other markers of inflammation and those involved in the destruction of articular cartilage in patients with PSA and the existence of mutual correlations and relationships between them. We found a statistically significant increase in serum levels of MMP-3, IL-6, COMP, and YKL-40 in the examined patients compared to the control group (Table 1). Similar results of studies by Chandran et al. also indicate that MMP-3 and COMP show increased serum activity in PsA patients and may be a sensitive marker differentiating PsA and Ps patients from healthy individuals, and MMP-3 itself is specific to PsA [44, 45]. In our study we also showed that the serum activity of COMP in PsA patients positively correlates with the number of swollen joints $(S J C)$ and the total joint count (TCJ + SJC; Table 2). Moreover, the COMP level in serum positively correlated with the degree of clinical severity expressed by the PASI and DLQI scale (Table 2). Similar conclusions were drawn by Skoumal et al., who studied serum COMP levels in patients with PsA and the cutaneous form of psoriasis [46]. They found that serum COMP levels in patients with PSA are clearly elevated and correlate with SJC.

From the results of our studies and observations, it can be concluded that also YKL-40 level in serum of pa-

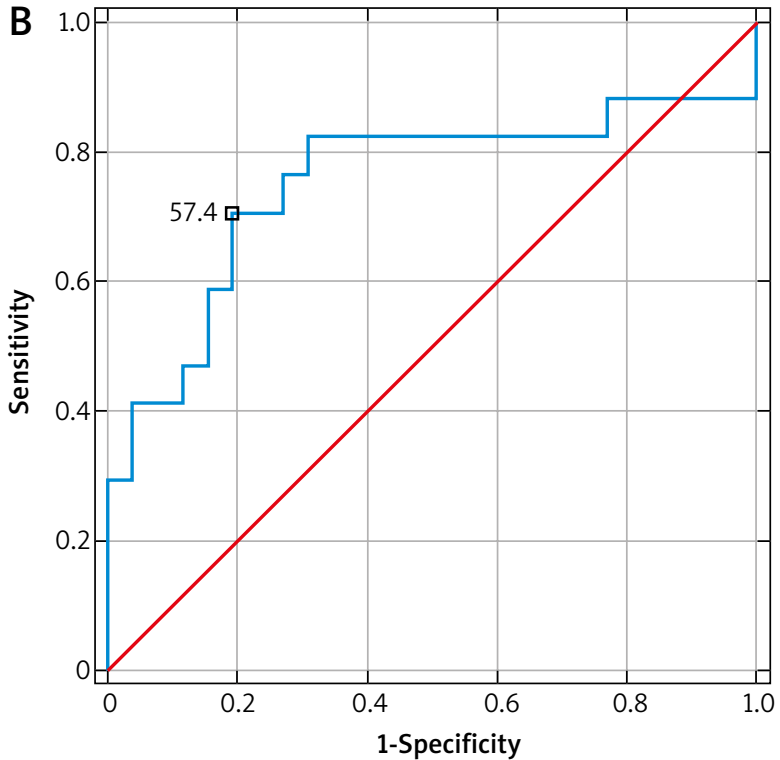

tients with PsA was clearly elevated in comparison with the control group. These results confirm previous reports on the role of this marker in the pathogenesis of PsA [47]. Jensen et al. noted that not only the YKL-40 serum level was elevated in patients with psoriasis, but also in patients with PsA the level of YKL-40 in serum was significantly higher than in patients with psoriasis cutaneous form (PsC). Our results confirm those of Ahmed et al., who observed that YKL-40 serum levels in patients with concomitant arthropathy (PSA) are higher than in patients with psoriasis alone [48].

We also decided to study whether each of studied biomarkers could serve as sensitive diagnostic tool in PsA screening (Figures 1-4; Table 3). It has been shown that COMP, as well as MMP-3, IL-18, and IL-20, could be the best candidates; however, more studies are needed to establish their role as PsA screening tools. We showed that COMP, MMP-3, and IL-18 could indicate the presence of PsA (cut-off value for COMP, MMP-3, IL-18: 1935.0 ng/ $\mathrm{ml} ; 14.30 \mathrm{ng} / \mathrm{ml} ; 40.18 \mathrm{pg} / \mathrm{ml}$, respectively). It means that patients without any radiographic or laboratory studies but with such values of these mediators independently could be suffering from PsA. These biomarkers need to be evaluated in the future in terms of overlapping syndromes and suspicion of PSA in patients without laboratory or radiographic imaging confirmation. The drawback of our results is the small study size, so our findings should be verified in further studies to provide a sensitive biomarker for PsA detection.

The results of our work may therefore confirm that the studied interleukins, in particular IL-18, may play an important role in the pathogenesis of psoriatic arthritis. We also found significantly increased serum activity of mediators responsible for the processes of degradation 
and destruction of articular cartilage (MMP-3, COMP, YKL-40). The above observations as well as numerous positive correlations of COMP with clinical indicators of disease progression in patients with PSA (Table 2), and the result of ROC curves analysis indicate that IL-18, COMP, YKL-40, and MMP-3 may be important biomarkers of PsA aetiopathogenesis. However, further studies and larger groups of patients are needed to correlate the interaction of these biomarkers and to confirm the direct effect of IL-18 on the processes of joint cartilage degradation in PSA. This, in turn, may give rise to the search for new therapeutic options for inhibition of biochemical processes induced and controlled by IL-18. The sense of such studies is confirmed by the reports of Smeets et al. [49]. In the collagen-induced model of osteoarthritis in mice they demonstrated that blocking the action of IL-18 through the administration of binding proteins results in a milder and less symptomatic course of arthrosis and destruction of articular cartilage [49].

However, our work has several weak points and limitations. The first of them is the relatively small group of patients. These studies, however, are preliminary, but they already indicate the advisability of their continuation. The authors of the publication continue their research by extending the group of patients qualified for analysis. It would be noteworthy to include in the study patients with the form of cutaneous psoriasis, in order to establish a panel of markers differentiating both forms of psoriasis for early detection of joint lesions in patients with psoriasis. Another limitation is the lack of randomisation of the study group. It also seems appropriate to analyse the tested biomarkers in serum and synovial fluid simultaneously. This would allow wider and more objective conclusions to be drawn. It is also advisable in the future to perform a wider range of immunoenzymatic studies with determination of activity of other anti-inflammatory markers (e.g. IL-4, IL-10, IL-13), other markers of joint cartilage and bone tissue destruction - crosslinked C-telopeptide of type I collagen (CTXI), cross-linked $\mathrm{N}$-telopeptide of type I collagen (NTXI), and N-terminal procollagen III propeptide (PIIINP).

\section{Conclusions}

The results show an increased concentration of IL-18 in serum of PsA patients, but its role in the aetiopathogenesis of psoriatic arthritis requires further research. It also seems that IL-18 and IL-20 as well as COMP and MMP-3 may be sensitive markers in the diagnosis of PsA.

\section{Acknowledgments}

The study was supported by internal funds of the Medical University of Lodz, Lodz, Poland (grant numbers: 502-03/7-048-02/502-54-006; 503/5-064-04/503-01 and 503/1-152-01/503-11-001-1900).

\section{Conflict of interest}

The authors declare no conflict of interest.

\section{References}

1. de Vlam K, Gottlieb AB, Mease PJ. Current concepts in psoriatic arthritis: pathogenesis and management. Acta Derm Venereol 2014; 94: 627-34.

2. Rahimi H, Ritchlin CT. Altered bone biology in psoriatic arthritis. Curr Rheumatol Rep 2012; 14: 349-57.

3. Chang CA, Gottlieb AB, Lizzul PF. Management of psoriatic arthritis from the view of the dermatologist. Nat Rev Rheumatol 2011; 7: 588-98.

4. Gottlieb AB, Mease PJ, Jackson JM, et al. Clinical characteristics of psoriatic arthritis and psoriasis in dermatologists' offices. J Dermatolog Treat 2006; 17: 279-87.

5. Bartosińska J, Zakrzewska E, Purkot J, et al. Decreased blood CD4+PD-1+ and CD8+PD-1+ T cells in psoriatic patients with and without arthritis. Adv Dermatol Allergol 2018; 35: 34450.

6. Cretu D, Prassas I, Saraon P, et al. Identification of psoriatic arthritis mediators in synovial fluid by quantitative mass spectrometry. Clin Proteomics 2014; 11: 27-7.

7. Coates LC, Kavanaugh A, Mease PJ, et al. Group for research and assessment of psoriasis and psoriatic arthritis 2015 treatment recommendations for psoriatic arthritis. Arthritis Rheumatol 2016; 68: 1060-71.

8. Scrivo R, Conigliaro P, Riccieri V, et al. Distribution of interleukin-10 family cytokines in serum and synovial fluid of patients with inflammatory arthritis reveals different contribution to systemic and joint inflammation. Clin Exp Immunol 2015; 179: 300-8.

9. Przepiera-Będzak H, Fischer K, Brzosko M. Extra-articular symptoms in constellation with selected serum cytokines and disease activity in spondyloarthritis. Mediators Inflamm 2016; 2016: 7617954.

10. Gracie JA, Forsey RJ, Chan WL, et al. A proinflammatory role for IL-18 in rheumatoid arthritis. J Clin Invest 1999; 104: 1393401.

11. Volin MV, Koch AE. Interleukin-18: a mediator of inflammation and angiogenesis in rheumatoid arthritis. J Interferon Cytokine Res 2011; 31: 745-51.

12. Dai S, Shan Z, Nishioka K, Yudoh K. Implication of interleukin18 in production of matrix metalloproteinases in articular chondrocytes in arthritis: direct effect on chondrocytes may not be pivotal. Ann Rheum Dis 2005; 64: 735-42.

13. Yamamura M, Kawashima M, Taniai M, et al. Interferongamma-inducing activity of interleukin-18 in the joint with rheumatoid arthritis. Arthritis Rheum 2001; 44: 275-85.

14. Olee T, Hashimoto S, Quach J, Lotz M. IL-18 is produced by articular chondrocytes and induces proinflammatory and catabolic responses. J Immunol 1999; 162: 1096-100.

15. Ruan G, Xu J, Wang K, et al. Associations between knee structural measures, circulating inflammatory factors and MMP13 in patients with knee osteoarthritis. Osteoarthritis Cartilage 2018; 26: 1063-9.

16. Blumberg H, Conklin D, Xu WF, et al. Interleukin 20: discovery, receptor identification, and role in epidermal function. Cell 2001; 104: 9-19.

17. Lebre MC, Jonckheere CL, Kraan MC, et al. Expression of IL20 in synovium and lesional skin of patients with psoriatic arthritis: differential response to alefacept treatment. Arthritis Res Ther 2012; 14: R200. doi: 10.1186/ar4038. 
18. Wei CC, Hsu YH, Li HH, et al. IL-20: biological functions and clinical implications. J Biomed Sci 2006; 13: 601-12.

19. Hsu YH, Yang YY, Huwang MH, et al. Anti-IL-20 monoclonal antibody inhibited inflammation and protected against cartilage destruction in murine models of osteoarthritis. PLoS One 2017; 12: e0175802.

20. Hsu YH, Chang MS. IL-20 in rheumatoid arthritis. Drug Discov Today 2015; 22: 960-4.

21. Hsu YH, Li HH, Hsieh MY, et al. Function of interleukin-20 as a proinflammatory molecule in rheumatoid and experimental arthritis. Arthritis Rheum 2006; 54: 2722-33.

22. Li HH, Hsu YH, Wei CC, et al. Interleukin-20 induced cell death in renal epithelial cells and was associated with acute renal failure. Genes Immun 2008; 9: 395-404.

23. Waszczykowska A, Goś R, Waszczykowska E, et al. Assessment of skin microcirculation by laser Doppler flowmetry in systemic sclerosis patients. Adv Dermatol Allergol 2014; 31: 6-11.

24. Hsu YH, Chang MS. Interleukin-20 antibody is a potential therapeutic agent for experimental arthritis. Arthritis Rheum 2010; 62: 3311-21.

25. Wei CC, Li HH, Hsu YH, et al. Interleukin-20 targets renal cells and is associated with chronic kidney disease. Biochem Biophys Res Commun 2008; 374: 448-53.

26. Tritsaris K, Myren M, Ditlev SB, et al. IL-20 is an arteriogenic cytokine that remodels collateral networks and improves functions of ischemic hind limbs. Proc Natl Acad Sci USA 2007; 104: 15364-9.

27. Chen WY, Cheng BC, Jiang MJ, et al. IL-20 is expressed in atherosclerosis plaques and promotes atherosclerosis in apolipoprotein E-deficient mice. Arterioscler Thromb Vasc Biol 2006; 26: 2090-5.

28. Wei CC, Chen WY, Wang YC, et al. Detection of IL-20 and its receptors on psoriatic skin. Clin Immunol 2005; 117: 65-72.

29. Stenderup K, Rosada C, Worsaae A, et al. Interleukin-20 as a target in psoriasis treatment. Ann N Y Acad Sci 2007; 1110: 368-81.

30. Stenderup K, Rosada C, Worsaae A, et al. Interleukin-20 plays a critical role in maintenance and development of psoriasis in the human xenograft transplantation model. Br J Dermatol 2009; 160: 284-96.

31. Otkjaer K, Kragballe K, Funding AT, et al. The dynamics of gene expression of interleukin-19 and interleukin-20 and their receptors in psoriasis. Br J Dermatol 2005; 153: 911-8.

32. Wang F, Lee E, Lowes MA, et al. Prominent production of IL-20 by CD68+/CD11c+ myeloid-derived cells in psoriasis: gene regulation and cellular effects. I Invest Dermatol 2006; 126: 1590-9.

33. Mease PJ. Measures of psoriatic arthritis. Arthritis Care Res (Hoboken) 2011; 63 Suppl 11: S64-85.

34. Long CC, Finlay AY. The finger-tip unit: a new practical measure. Clin Exp Dermatol 1991; 16: 444-7.

35. Ashcroft DM, Wan Po AL, Williams HC, Griffiths CE. Clinical measures of disease severity and outcome in psoriasis: a critical appraisal of their quality. Br J Dermatol 1999; 141: 185-91.

36. Finlay AY, Khan GK. Dermatology Life Quality Index (DLQI): a simple practical measure for routine clinical use. Clin Exp Dermatol 1994; 19: 210-6.

37. Taylor W, Gladman D, Helliwell P, et al. Classification criteria for psoriatic arthritis: development of new criteria from a large international study. Arthritis Rheum 2006; 54: 2665-73.

38. Gladman DD, Mease PJ, Strand V, et al. Consensus on a core set of domains for psoriatic arthritis. J Rheumatol 2007; 34: 1167-70.
39. Kragstrup TW, Otkjaer K, Holm C, et al. The expression of IL-20 and IL-24 and their shared receptors are increased in rheumatoid arthritis and spondyloarthropathy. Cytokine 2008; 41: 16-23.

40. Kragstrup TW, Greisen SR, Nielsen MA, et al. The interleukin-20 receptor axis in early rheumatoid arthritis: novel links between disease-associated autoantibodies and radiographic progression. Arthritis Res Ther 2016; 18: 61.

41. Rooney T, Murphy E, Benito M, et al. Synovial tissue interleukin-18 expression and the response to treatment in patients with inflammatory arthritis. Ann Rheum Dis 2004; 63: 1393-8.

42. Przepiera-Będzak H, Fischer K, Brzosko M. Serum interleukin-18, fetuin-A, soluble intercellular adhesion molecule-1, and endothelin-1 in ankylosing spondylitis, psoriatic arthritis, and SAPHO syndrome. Int J Mol Sci 2016; 17: 1255.

43. Bresnihan B, Roux-Lombard P, Murphy E, et al. Serum interleukin 18 and interleukin 18 binding protein in rheumatoid arthritis. Ann Rheum Dis 2002; 61: 726-9.

44. Chandran V, Cook RJ, Edwin J, et al. Soluble biomarkers differentiate patients with psoriatic arthritis from those with psoriasis without arthritis. Rheumatology 2010; 49: 1399405.

45. Chandran V. Soluble biomarkers may differentiate psoriasis from psoriatic arthritis. J Rheumatol Suppl 2012; 89: 65-6.

46. Skoumal M, Haberhauer G, Fink A, et al. Increased serum levels of cartilage oligomeric matrix protein in patients with psoriasis vulgaris: a marker for unknown peripheral joint involvement? Clin Exp Rheumatol 2008; 26: 1087-90.

47. Jensen $P$, Wiell C, Milting K, et al. Plasma YKL-40: a potential biomarker for psoriatic arthritis? J Eur Acad Dermatol Venereol 2013; 27: 815-9.

48. Ahmed SF, Attia EA, Saad AA, et al. Serum YKL-40 in psoriasis with and without arthritis; correlation with disease activity and high resolution power Doppler ultrasonographic joint findings. J Eur Acad Dermatol Venereol 2015; 29: 682-8.

49. Smeets RL, van de Loo FA, Arntz OJ, et al. Adenoviral delivery of IL-18 binding protein C ameliorates collagen-induced arthritis in mice. Gene Ther 2003; 10: 1004-11. 